Z. klin. Chem. u. klin. Biochem.

8. Jg., S. 134-136, März 1970

\title{
Eine neue Methode zur photometrischen Bestimmung der Triglyceride (Neutralfette)
}

\author{
Von J. Hoeflmayr ${ }^{1}$ ) und R. Fried \\ unter technischer Mitarbeit von A. Cordua und G. Knecht \\ Aus den wissenscbaftlicben Laboratorien der Firma Dr. Heinz Haury, Mïnchen 23
}

(Eingegangen am 9. Oktober 1969)

Bei der beschriebenen Methode zur Bestimmung der Triglyceride werden nach Fällung des freien Cholesterins und nach Adsorption der Phosphatide an Kieselgel die restlichen Esterbindungen nach der Hydroxamsäuremethode und das veresterte Cholesterin nach der LreBERMANN-BurChard-Reaktion jeweils in mVal/l ermittelt. Die Differenz stellt den Wert der Triglyceride dar. Einzelheiten der Bedingungen und die Durchführung werden beschrieben. Kritische Anmerkungen befassen sich mit der Fehlerbreite, der Genauigkeit (Variationskoeffizient) und der Korrelation zur enzymatischen Triglyceridbestimmung.

A new method for the photometric determination of triglycerides (neutral fats)

A method is described for the determination of triglycerides. After precipitation of the free cholesterol and adsorption of the phosphatides on kieselgel, the remaining total esters are determined by the hydroxamic acid method and the cholesterol esters by the LIEBERMANNBurchard reaction, both in meq/l. The difference represents the triglycerides. Details of the conditions and procedure are given. The error, the accuracy (variation coefficient) and correlation with the enzymic determination of triglycerides are critically discussed.

Die Bestimmung der Triglyceride sollte zur differentialdiagnostischen Abklärung von Fettstoffwechselstörungen in jedem Fall durchgeführt werden. Wenn dies unterlassen wurde, so liegt das an den Schwierigkeiten, welche die bisher bekannten Methoden im Routinelaboratorium mit sich bringen.

Zwei Methoden werden im allgemeinen für die direkte Bestimmung der Neutralfette angewendet. Nach der Methode von CARISON und WADSTRÖM (1) werden die Triglyceride im Serum nach einer Reihe von acht Arbeitsschritten als Formaldehyd, der durch Oxydation von Glycerin entstanden ist, mittels Chromotropsäure als Farbreagenz bestimmt. Die zahlreichen Arbeitsschritte und die schlechte Haltbarkeit des Farbreagenz sind ein großer Nachteil dieser Bestimmungsmethode. Eine andere, sehr elegante Methode wurde von EGGSTEIN und KREUTZ (2) angegeben. Sie beruht auf drei gleichzeitig im Bestimmungsansatz ablaufenden Fermentreaktionen, deren spezifisches Substrat in der ersten Stufe das Glycerin ist und deren dritte Stufe die NADH verbrauchende LDH-Reaktion ist. Zwar sind hier nur drei Arbeitsschritte notwendig, was gegenüber der CARLSON-Methode einen Fortschritt bedeutet, doch erfordert die Herstellung und exakte Einstellung der zahlreichen Reagenzlösungen einen großen Arbeitsaufwand. Die Haltbarkeit der Fermentlösungen ist teilweise auch bei Aufbewahrung im Kühlschrank auf wenige Tage begrenzt. Für die Durchführung der Bestimmung müssen besondere Anforderungen an Sorgfalt und Erfahrung gestellt werden, die im Routinelaboratorium nicht leicht erreicht werden.

Unsere Methode geht von folgender Überlegung aus: Gelingt es, aus einem Serum das freie Cholesterin und

$\left.{ }^{1}\right)$ Teilwreise vorgetragen auf der 13. Jahresversammlung der Schweizerischen Vereinigung für Klinische Chemie, 18.-20. April 1969 in Lugano, Kurzreferat Schweiz. med. Wschr. 99, 1826 (1969). die Phosphatide zu entfernen, so finden sich im verbleibenden Reaktionsansatz nur noch die Triglyceride einschließlich der Mono- und Diglyceride und das veresterte Cholesterin. Bestimmt man nun die GesamtEsterbindungen und die des veresterten Cholesterins in $\mathrm{mVal} / l$, so muß die Differenz der beiden den Esterbindungen der Triglyceride ebenfalls in $\mathrm{mVal} / l$ entsprechen.

Voraussetzung für die Realisierung war, ein Lösungsmittel zu finden, das die vollständige quantitative Adsorption der Phosphatide an Kieselgel gewährleistet und gleichzeitig die Bestimmung der Esterbindungen nach der Hydroxamsäuremethode (4) sowie die des veresterten Cholesterins nach der LIEBERMANN-BURCHARD-Reaktion (5) zuläßt. Das von CARLson verwendete Chloroform kommt hierfür nicht in Frage, da der sich im alkalischen Milieu daraus bildende Orthoameisensäureester die Hydroxamsäuremethode stört. Dagegen erwies sich eine Mischung von wasserfreiem Isopropanol und sek. Butanol im Verhältnis $1: 2(\mathrm{v} / \mathrm{v})$ als geeignet.

\section{Reagenzien $\left.{ }^{2}\right)$}

1. Digitoninlösung (0,25proz. in wassetfreiem Isopropanol)

2. Digitonin-Cholesterin-Standard, entsprechend $5,2 \mathrm{mVal} / \mathrm{l}(0,4 \mathrm{~g}$ Cholesterin werden in $1 /$ einer 0,25 proz. Digitoninlösung in Isopropanol gelöst)

3. Cholesterin-Reagens (0,05M 2,5-Dimethyl-benzol-sulfonsäure in einem Gemisch aus $300 \mathrm{ml}$ Essigsäureanhydrid und $200 \mathrm{ml}$ Eisessig)

4. Sek. Butylalkohol

5. Adsorptionsmittel (100 mg aktiviertes Kieselgel pro Kapsel) 6. Hydroxylaminhydrochloridlösung (11proz. in Methanol)

2) Haury-Test „Triglyceridec Dr. H. Haury, Chemische Fabrik, München 23, Postfach 48. 
7. Triolein-Standard, entsprechend $17 \mathrm{mVal} / \mathrm{l}$ Triolein $(0,334 \mathrm{~g}$ Triolcin werden in $0,334 / 0,5$ proz. Digitoninlösung in Isopropanol gegeben und mit 0,66 l sek. Butanol aufgefüllt)

8. 10proz. Eisen[III]-chloridlösung in 0,1N Salzsäurc

9. 10proz. Natronlauge

10: Säzsäure, mindestens $3,8 \mathrm{~N}$

11. Konzentrierte Schwefelsäure.

\section{Methodik}

\section{Entfernung des freien Cholesterins}

$0,5 \mathrm{ml}$ Serum werden mit $2,0 \mathrm{ml}$ Digitoninlösưng versetzt. Der Ansatz wird kurz aufgeschüttelt und bleibt anschließend $10 \mathrm{Min}$. stehen. Nach dem Zentrifugieren und Abgießen in ein trockenes Reagenzglas erhält man den Uberstand I. Von diesem Uberstand I werden $1 \mathrm{~m} /$ zur Eliminierung der Phosphatide und anschließenden Bestimmung der restlichen Esterbindungen und später $0,2 \mathrm{ml}$ zur Bestimmung des veresterten Cholesterins eingesetzt.

\section{Entfernung der Phospbatide}

In ein trockenes Reagenzglas werden $200 \mathrm{mg}$ Kieselgel (Inhalt von 2 Kapseln) gegeben, dazu $1,0 \mathrm{ml}$ vom Uberstand I und daran anschließend $2,0 \mathrm{~m} / \mathrm{sek}$. Butylalkohol. Ein gleichfalls angesetzter Leerwert enthält $1,0 \mathrm{~m} l$ sek. Butylalkohol und $0,5 \mathrm{~m} l$ Digitoninlösung. Während der Leerwert nur gut gemischt werden muß, wird die Analyse $5 \mathrm{Min}$. kräftig geschüttelt. Nach dem Abzentrifugieren des Analysenansatzes erhält man den Uberstand II. Es ist besonders darauf zu achten, daß vom Adsorptionsmittel nichts in den Überstand gebracht wird.

\section{Bestimmung der restlichen Esterbindungen}

In drei neue vorbereitete Reagenzgläser werden nacheinander für Analyse, Standard und Leerwert einpipettiert: Je $0,5 \mathrm{ml}$ einer 10proz. Natronlauge und anschließend je $0,5 \mathrm{ml}$ Hydroxylaminhydrochlorid-Lösung. In den Analysenansatz werden $1,0 \mathrm{~m} /$ des Uberstandes II, in den Standard $1,0 \mathrm{~m} /$ des Triolein-Standards und in dem Leerwert $1,0 \mathrm{ml}$ des bei Entfernung der Phosphatide erhaltenen Leerwertgemisches gegeben. Alle Ansätze bleiben nunmehr $60 \mathrm{Min}$. bei Raumtemperatur stehen. Danach werden in alle drei Röhrchen je $0,5 \mathrm{ml} 3,8 \mathrm{~N}$ Salzsäure und $0,5 \mathrm{ml}$ Eisen[III]-chloridlösung pipettiert. Nach dem Mischen und nach einer Pause von $20 \mathrm{Min}$. werden Standard und Analyse gegen den Leerwert bei einer Wellenlänge zwischen 500 und $550 \mathrm{~nm}$ gemessen.

\section{Für die Berecbnung gilt die Gleichung}

$$
\frac{\text { Extinktion Analyse }}{\text { Extinktion Standard }} \cdot 17=\mathrm{mVal} / / \text { Esterbindungen }
$$

\section{Bestimmung des veresterten Cholesterins}

In drei vorbereitete Reagenzgläser werden jeweils pipettiert: $2,0 \mathrm{ml}$ Cholesterinreagenz. In den Analysenansatz werden $0,2 \mathrm{ml}$ des Überstandes $\mathrm{I}$, in den Standardansatz $0,2 \mathrm{ml}$ DigitoninCholesterin-Standard und in den Leerwert $0,2 \mathrm{ml}$ Digitoninlösung gegeben. Die Proben, die sich leicht erwärmen, bleiben 10 Min. bei Raumtemperatur stehen und werden anschließend mit jeweils $0,3 \mathrm{~m} l$ konz. Schwefelsäure versetzt. Unter neuerlichem Erwärmen bleiben die Proben 10-15 Min. bei diffusem Tageslicht stehen. Danach werden Analyse und Standard gegen den Leerwert bei einer Wellenlänge zwischen 560 und $590 \mathrm{~nm}$ gemessen. Der Geradlinigkeit der Eichkurve entsprechend gilt die Berechnungsgleichung:

\section{$\frac{\text { Extinktion Analyse }}{\text { Extinktion Standard }} \cdot 5,2=\mathrm{mVal} / \mathrm{l}$ verestertes Cholesterin}

Berechnung der Triglyceride: $\mathrm{mVal} / \mathrm{l}$ Gesamt-Esterbindungen $\mathrm{mVal} / /$ verestertes Cholesterin $=\mathrm{mVal} / /$ Triglyceride.

\section{Kritik der Methode}

1. Um die Frage zu klären, ob das veresterte Cholesterin auch tatsächlich im richtigen Bereich erfaßt wird, wurden eine Reihe von Seren in getrennten Arbeitsgängen auf das Gesamt-Cholesterin und auf das veresterte und freie Cholesterin untersucht. Die Summe von freiem und verestertem Cholesterin war im Bereich der Fehlerbreite der Methode avon $\pm 3 \%$ gleich dem Gehalt an Gesamt-Cholesterin.

2. Die unterschiedlichen Absorptionsmaxima von Digitonin und Cholesterin bei Wellenlängen über $550 \mathrm{~nm}$ sowie das Mitführen eines Digitonin-Cholesterin-Standards schließen einen durch den Digitoningehalt der Analyse entstehenden Fehler aus, worauf früher schon hingewiesen wurde $(6,7)$.

3. Nach der Behandlung mit aktiviertem Kieselgel darf der verbleibende Ansatz keine Phosphatide mehr ent. halten. Wir konnten dann keine Phosphatide im Überstand nachweisen, wenn der Reaktionsansatz nach $\mathrm{Zu}-$ gabe des Adsorptionsmittels $5 \mathrm{Min}$. geschüttelt worden war. Die einfache Zugabe des Adsorptionsmittels genügt also nicht.

4. Der Einwand von Eggstern (3), daß die Hydroxamsäurebildung der Fettsäure-Cholesterinester nur in absolut wasserfreiem Milieu erfolgt, während im teilweise wäßrigen Milieu eine solche Reaktion nicht stattfindet, legt den Gedanken nahe, Reaktionsbedingungen zu suchen, bei denen nur die Triglyceride und nicht die Cholesterinester zu den Hydroxamsäuren verseift werden. Man könnte dann nach Entfernung der Phosphatide auf einfache Weise die Triglyceride bestimmen. Dies war leider nicht möglich. Im Gegensatz zu EGGSTEIN, der seine Versuche mit Cholesterinpalmitat und -stearat und unter anderen Einwirkungszeiten durchgeführt hat, konnten wir feststellen, daß unter den von uns gewählten Versuchsbedingungen Cholesterinester von ungesättigten Fettsäuren zu $99-100 \%$ und von gesättigten Fettsäuren zu $75 \%$ zu Hydroxamsäuren verseift werden. Nun machen die gesättigten Fettsäure-Cholesterinester etwa $20 \%$ der gesamten Cholesterinester aus, so daß bei der Triglyceridbestimmung die Werte um höchstens $5 \%$ niedriger zu erwarten sind.

Wenn man die in der Tabelle 1 aufgeführten Wertepaare addiert, so zeigt sich, daß die mit der angegebenen

Tab. 1

Photometrische Bestimmung der Triglyceride. Vergleich der beschriebenen mit der enzymatischen Methode

\begin{tabular}{|c|c|c|c|}
\hline \multirow[b]{2}{*}{ Serum Nr. } & \multicolumn{3}{|c|}{ Triglyceride [mVal/l] } \\
\hline & $\begin{array}{l}\text { Beschriebene } \\
\text { Methode }\end{array}$ & $\begin{array}{l}\text { Methode nach } \\
\text { EGGSTEIN (2) }\end{array}$ & $\begin{array}{l}\text { Angegebener } \\
\text { Wert }\end{array}$ \\
\hline $\begin{array}{l}\text { 1. } \\
\text { 3. } \\
\text { 4. } \\
\text { 5. } \\
6 . \\
\text { 7. } \\
\text { 8. } \\
\text { 10. Moni-Trol I } \\
11 . \text { Moni-Trol II } \\
\text { 12. Hyland-Spezial } \\
\text { 13. Hyland-Spezial } \\
\text { 14. Hyland-Spezial }\end{array}$ & $\begin{array}{r}192,6 \\
310,5 \\
82,0 \\
100,1 \\
134,5 \\
111,2 \\
86,7 \\
227,0 \\
127,0 \\
155,0 \\
109,0 \\
73,0 \\
70,2 \\
72,0\end{array}$ & $\begin{array}{r}205,0 \\
274,0 \\
78,6 \\
113,8 \\
131,3 \\
121,0 \\
96,2 \\
259,0 \\
149,0 \\
156,0 \\
110,0 \\
69 \\
87,4 \\
\end{array}$ & $\begin{array}{c}81 \\
50 \\
75(65-85) \\
75 \\
70\end{array}$ \\
\hline
\end{tabular}


Methode gefundenen Ergebnisse im Durchschnitt nur um 3,8\% niedriger als die der Vergleichsmethode liegen. Der Korrelationskoeffizient zur Methode von EGgstern errechnet sich mit $r=0,964$.

5. Die Reproduzierbarkeit der beschriebenen Methode wurde durch die Berechnung des Variationskoeffizienten $(\mathrm{VK})^{3}$ ) geprüft. Zugrunde liegen dem VK die Ergebnisse von fünf verschiedenen Seren, an denen je 10 Untersuchungen vorgenommen wurden. Da zwei Ar-

3) $\mathrm{VK}=$ mittlerer Fehler der Einzelmessung fm ausgedrückt in $\%$ des Mittelwertes: $\mathrm{fm}=\sqrt{\frac{\sum(\mathrm{f})^{2}}{\mathrm{n}-1}}$. beitsschritte notwendig sind, müssen jedoch zwei VK ermittelt werden. Der VK für die Bestimmung des veresterten Cholesterins beträgt 2,66, der für die restlichen Esterbindungen 2,14. Da sich die Fehler addieren können, ist der VK für die Gesamtbestimmung im ungünstigsten Fall mit 4,8 anzusetzen.

6. Vielfach wird der Wert nicht in $\mathrm{mVal} / l$, sondern in $\mathrm{mg} / 100 \mathrm{ml}$ gewünscht. Bezieht 'man das Ergebnis auf Triolein, so müssen die $\mathrm{mVal} / \mathrm{l}$ mit 29,5 multipliziert werden. Wird jedoch auf Triglyceride mit einem durchschnittlichen Molekulargewicht der Serumfettsäuren von 275 bezogen, so ist der Faktor 28,5 einzusetzen.

\section{Literatur}

1. Carlson, L. A. und L. B. Wadström, Clin. chim. Acta Amsterdam 4, 197 (1959). - 2. EggsteIn, M. und F. H. Kreutz, Klin. Wschr. 44: 262 (1966). - 3. Eggstein, M., diese Z. 4, 12 (1966). 4. Fried, R. und J. Hoeflasayr, Klin. Wschr. 41, 727 (1963).
5. Fried, R. und J. HoeflumaYR, Med: Klin. 64, 1320 (1969). 6. Hoefluayr, J. und R. Fried, Med. Welt, 2015 (1964). 7. Hoeflamar, J. und R. Fried, Ergebn. Labor.-Med. 2, 119 (1965).
Dr. med. Joachim Hoeflmayr Dr. rer. nat. Rudolf Fried 8 München 13

Schleißheimer Str. 343 